\title{
WET SEASON SPATIAL OCCURRENCE OF PHYTOPLANKTON AND ZOOPLANKTON IN LAGOS LAGOON, NIGERIA.
}

\author{
${ }^{*}$ NKWOJI, J. A., ONYEMA I. C. ${ }^{2} \&$ IGBO, J. K. ${ }^{1}$ \\ 1Department of Biological Oceanography, Nigerian Institute for \\ Oceanography and Marine Research, Lagos, Nigeria. \\ ${ }^{2}$ Department of Marine Sciences, University of Lagos, Akoka, \\ Lagos, Nigeria. \\ *iosephniomr@yahoo.com
}

\begin{abstract}
Investigation into the spatial occurrence of wet season phytoplankton and zooplankton in Lagos lagoon, Nigeria was carried out in October, 2008 in 12 stations. A total of 36 species of phytoplankton from 21 genera, 20 zooplankton species from 17 genera and 10 juvenile forms were recorded for the study. The results showed that freshwater conditions within the lagoon were due to high freshwater inflow from adjoining waterways and reduced tidal incursion from the sea. The wet season spectrum of the lagoon was dominated by diatoms for the phytoplankton and copepods for the zooplankton. The presence and array of juvenile forms in the wet season suggest that the lagoon remain a sanctuary, nursery and breeding grounds for aquatic species Crustaceans dominated the spectrum of the juvenile forms.
\end{abstract}

Keywords: Plankton, Physico-chemical characteristics, lagoon, juvenile forms, Lagos.

\section{INTRODUCTION}

An intricate network of creeks, rivers and lagoons exist in southwestern Nigeria which eventually connects to the sea via the Lagos harbour (Onyema, 2007). Lagoons in this region are connected and run parallel to the Gulf of Guinea coastline over a distance of 237 $\mathrm{km}$ (Hill \& Webb, 1958). The Lagos lagoon is open and tidal. The lagoon, with a surface area of $208 \mathrm{~km}^{2}$ and an average depth of $1.5 \mathrm{~m}$ is a shallow micro-tidal environment (FAO, 1969). Salinity regime in the lagoon is seasonal with high salinities reported from December to April and low salinities observed between May and November. Various ecologists (Hill \& Webb, 1958; Olaniyan, 1975; Nwankwo, 1986) have attributed salinity gradients within the lagoon to two main factors; influx of floodwater from rivers and creeks surrounding wetlands and tidal seawater inflow through the Lagos harbour.

Investigations of anthropogenic wastes and environmental modifications in the Lagos lagoon have revealed increased levels of pollution stress (Ajao,1996, Onyema et al., 2003, 2007; Edokpayi \& Nkwoji, 2007). According to Nwankwo (2004), an important ecological ramification of increasing population pressure, poor sewerage system, industrialization and poor waste management in Nigerian's coastal area is that pollutants freely find their way unabated into our coastal waters through drains, canals, rivers, creeks and lagoons that act as conduits. Apart from enriching the water with high amounts of biodegradable matter, these discharges introduces nutrients, toxic and other land based substances that may consequently signal epidemiological problems and an increase in human induced stressors which impairs aquatic biodiversity (Nwankwo \& Akinsoji, 1988).

Information dealing with the plankton species of the Lagos lagoon and its environs is quite scanty. Existing reports include Olaniyan (1969) on the plankton of the lagoons of South-Western Nigeria and Akpata et al., (1993) on the effects of organic pollution on plankton and benthic population of parts of the Lagos lagoon. More recent studies include Onyema et al., $(2003,2007)$ at point sources of pollution, Nwankwo et al., (2008) on the Kuramo lagoon, Emmanuel \& Onyema (2007) on a tidal creek (Abule-elude creek) and Onyema \&Ojo (2008) on the lower Ogun river (Agboyi creek). The aim of this work is to investigate the spatial occurrence of phytoplankton and zooplankton in the wet season in relation to environmental characteristics.

\section{MATERIALS AND METHODS}

The Study Area: The Lagos lagoon (Fig. 1) located in Lagos State, Nigeria and is one of the nine lagoons in South-western Nigeria (Webb, 1958a; Nwankwo, 2004b; Onyema, 2008a). The lagoon is an open, shallow and tidal lagoon, with a surface area of $208 \mathrm{~km}^{2}$ (FAO, 1969) and an average depth of less than two meters. It provides the only opening to the sea for the nine lagoons of South Western Nigeria. Owing to the dynamics of river inflow and seawater incursion, the Lagos lagoon experiences brackish condition that is more discernable in the dry season. In the wet season, the increased river inflow creates freshwater and low brackish conditions in various parts of the lagoon. The harmattan, a short season of dry, dusty North-East Trade winds are experienced sometimes between November and January in the region reducing visibility and lowering temperatures (Onyema et al., 2003).

In the Lagos lagoon, there is a direct relation between the seasonal bimodal rainfall pattern, the environmental gradient and the biotal gradient. South-western Nigeria is endowed with an intricate network of rivers, creeks and lagoons, which serve as conduits transferring highly nutrified waters from hinterland to coastal areas. Flood waters associated with rainfall are known to enrich the coastal environment, dilutes its ionic concentration and break down existing environmental gradients (Olaniyan, 1969; Nwankwo, 1996). Conversely in the dry season, freshwater inflow is greatly reduced and seawater enters the lagoon through the harbour giving rise to marine conditions near the harbour and brackish water extending far inland (Hill \& Webb, 1958; Nwankwo, 1996; Onyema et al., 2003). Hence, areas located in close proximity to the harbour experience greater marine influence than places further inland.

Collection and Analysis of Water Samples: Surface water samples were collected with a $1 \mathrm{dm}^{3}$ water sampler and stored in 1 litre water bottles and analysed in the laboratory for $\mathrm{pH}$, conductivity, salinity and turbidity using a multi-meter water checker (Horiba U-12). Separate water samples were collected in $250 \mathrm{ml}$ dissolved oxygen bottles at each station for dissolved oxygen estimation using iodometric Winkler's method. Air and surface water temperature were measured in situ using mercury-in-glass thermometers. Alkalinity of the water samples was determined by titrating dilute $\mathrm{HCl}$ against $50 \mathrm{ml}$ of the water sample using methyl orange as an indicator.

Collection and Analysis of Plankton Samples: Plankton sample was collected on each occasion with a $55 \mu \mathrm{m}$ mesh size standard plankton net held against the current of the ebbing tide for $10 \mathrm{mins}$. The net was then hauled in and the sample transferred to a $250 \mathrm{ml}$ well labelled plastic container with screw cap and preserved with 4\% unbuffered formalin and stored in the laboratory prior to microscopic analysis in the laboratory. 


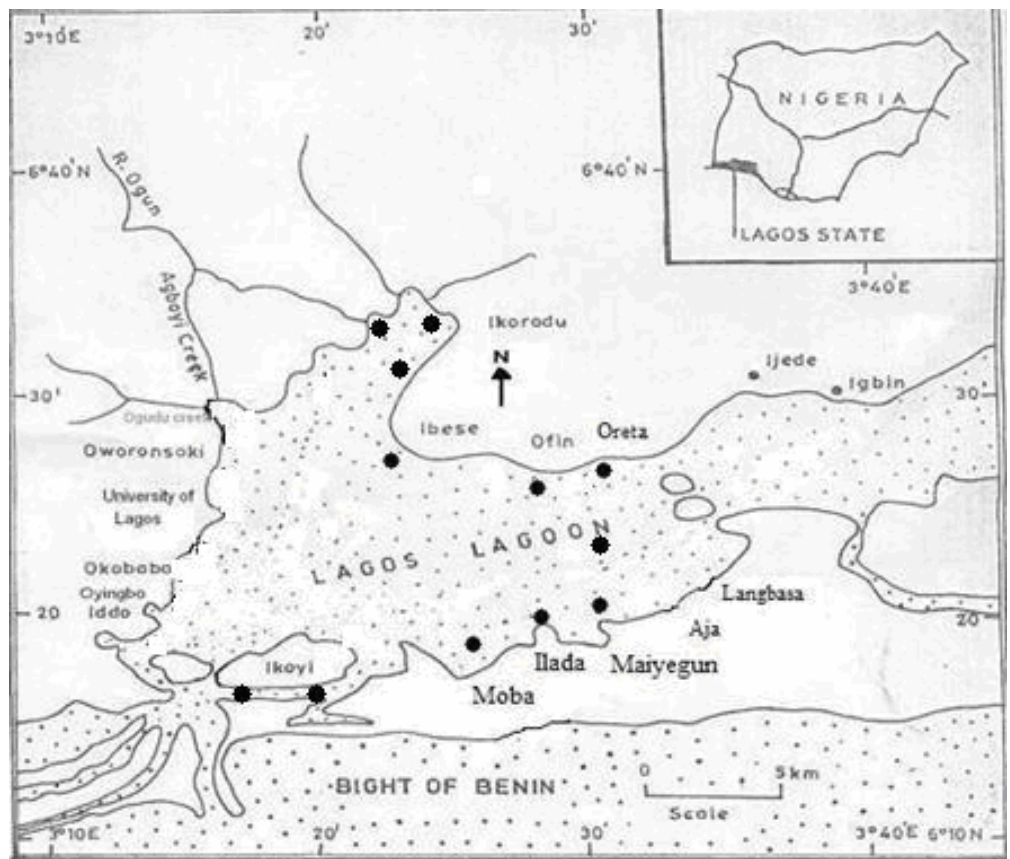

\section{FIG 1. MAP OF LAGOS LAGOON SHOWING THE SAMPLING STATIONS}

Ten $\mathrm{ml}$ of the concentrated sample was investigated for plankton analyses, at different magnifications (50X,100X and 400X) using a Wild II Binocular Microscope with calibrated eye piece and the average recorded. A suitable plankton sample mount was then created. The drop count microscope analysis method described by Onyema (2007) was used to estimate the plankton flora and fauna. Since each sample drop from the dropper accounts to $0.1 \mathrm{ml}$, the results on abundance / occurrence were multiplied accordingly to give the values as numbers of organisms per $\mathrm{ml}$ which is the standard unit of measurement. Organisms were observed for phytoplankton (cells, filaments, colonies) and zooplankton species (adults and juvenile stages alike). Final data were presented as number of organisms (cells, filaments, colonies and whole organism) per ml. Appropriate texts were used to aid identification of the species encountered (Wimpenny, 1966; Olaniyan, 1975; Vanlandingham, 1982; Nwankwo, 1990, 1995, 2004; Siver, 2003; Rosowski, 2003; Barnes et al., 1993 and Waife \& Frid, 2001).

Community Structure Analysis:

Species Richness Index (d): The Species richness index (d) was used to evaluate the community structure.

$$
d=\frac{S-1}{\ln N}
$$

Where:

$d=$ Species richness index

$\mathrm{S}=$ Number of species in a population

$\mathrm{N}=$ Total number of individuals in $\mathrm{S}$ species

Shannon and Wiener diversity index (Hs) (Ogbeibu, 2005). The Shannon and Wiener diversity index (Hs)

$$
H s=\frac{N \log N-\Sigma P i \log P i}{N}
$$

Where

$\mathrm{Hs}=\quad$ Shannon and Wiener diversity Index $\mathrm{i}=$ Counts denoting the ith species ranging from $1-n$
$\mathrm{Pi}=$ Proportion that the ith species represents in terms of numbers of individuals with respect to the total number of individuals in the sampling space as whole.

Species Equitability or Evenness index (j) (Ogbeibu, 2005). The Species Equitability or Evenness index (j)

$$
J=\frac{H s}{\log _{2} S}
$$

Where

$\mathrm{J}=$ Equitability index

$\mathrm{Hs}=$ Shannon and Weiner index

$\mathrm{S}=$ Number of species in a population

Simpsons dominance index (C) (Ogbeibu, 2005).

$$
C=\sum\left(\frac{n_{i}}{N}\right)^{2}
$$

Where

$n=$ the total number of organisms of a particular species

$\mathrm{N}=$ the total number of organisms of all species

\section{RESULTS}

The result of the physico-chemical parameters is shown in Table 1 while results of the phytoplankton, zooplankton and juvenile forms are presented in Tables $2 \mathrm{a}, 3 \mathrm{a}$ and $4 \mathrm{a}$ respectively with their community composition parameters (Table $2 b, 4 b$ and $4 b$ ). A total of 36 species of phytoplankton from 21 genera and 20 zooplankton species from 17 genera were recorded during the studies. Generally, stations within the Five Cowries creek recorded known brackish water species than species found in other parts of the lagoon. Furthermore the staition at Oreta recorded no zooplankton species for the study. A total of 10 juvenile forms were also recorded for the study. 
TABLE 1. PHYSICO-CHEMICAL PARAMETERS OF THE WATER SAMPLES

\begin{tabular}{|c|c|c|c|c|c|c|c|c|c|c|c|c|}
\hline & 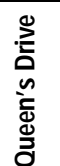 & 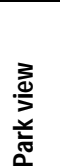 & $\frac{\pi}{0}$ & 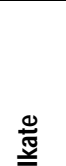 & 을 & 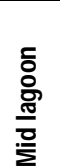 & 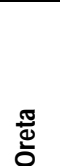 & 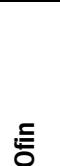 & $\begin{array}{l}\Phi \\
\Phi\end{array}$ & 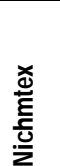 & $\begin{array}{l}\text { 늠 } \\
\text { 믐 } \\
\text { 흠 } \\
\end{array}$ & 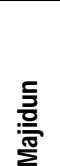 \\
\hline Air Temp. $\left({ }^{\circ} \mathrm{C}\right)$ & 28 & 27.2 & 26 & 28 & 30 & 30 & 30 & 30 & 30.5 & 30 & 30 & 31 \\
\hline $\mathrm{H}_{2} \mathrm{OTemp}$. ( $\left.{ }^{\circ} \mathrm{C}\right)$ & 29 & 29 & 28 & 28 & 28.9 & 29 & 29 & 29 & 29.5 & 28 & 28 & 28 \\
\hline $\mathrm{pH}$ & 9.1 & 8.9 & 9.0 & 9.1 & 8.9 & 8.8 & 9.2 & 8.9 & 9.0 & 9.0 & 8.8 & 9.1 \\
\hline $\begin{array}{l}\text { Conductivity } \\
\left(\mathrm{mScm}^{-1}\right)\end{array}$ & 0.84 & 1.0 & 0.64 & 0.67 & 0.61 & 0.12 & 0.19 & 0.3 & 0.24 & 0.54 & 0.18 & 0.61 \\
\hline Turbidity (NTU) & 78 & 366 & 126 & 108 & 208 & 226 & 86 & 386 & 248 & 256 & 346 & 276 \\
\hline Salinity $(\%$ & 0 & 0 & 0 & 0 & 0 & 0 & 0 & 0 & 0 & 0 & 0 & 0 \\
\hline D.O $\left(\mathrm{mgl}^{-1}\right)$ & 11.2 & 13.6 & 18.4 & 15.6 & 12.0 & 11.2 & 12.4 & 12.4 & 12.8 & 9.2 & 8.0 & 5.2 \\
\hline Alkalinity & 8.0 & 8.0 & 6.0 & 8.0 & 10.0 & 8.0 & 8.0 & 8.0 & 8.0 & 8.0 & 12.0 & 8.0 \\
\hline
\end{tabular}

Phytoplankton community structure: The species composition of the phytoplankton is presented in Table $2 \mathrm{a}$.

For the twelve stations studied, diversity (S) ranged between 5 species (Park view and Oreta) and 26 species (Nichemtex). Abundance of individuals per $\mathrm{ml}(\mathrm{N})$ of the samples was between 85 individuals per $\mathrm{ml}$ (Itedo) and 895 individuals per $\mathrm{ml}$ (Majidun). Whereas Log of Species diversity (Log S) ranged between 0.70 (Park view) and 1.04 (Ofin), Log of phytoplankton abundance (Log
$\mathrm{N})$ were between 1.93 (Itedo) and 2.95 (Majidun). Furthermore, the values for Shannon-Wiener Index (Hs) were between 0.48 (Park view) and 1.10 (Nichemtex). Menhinick Index (D) on the other hand ranged between 0.48 (Park view) and 1.09 (Nichemtex). Margalef Index (d) values also ranged between 0.82 (Oreta) and 3.68 (Majidun). Equitability Index (j) index values were between 0.57 (Ikorodu port) and 0.93 (Queens Drive). Simpson's Dominance Index (C) also ranged between 0.09 (Nichemtex) and 0.43 (Oreta). (Table2b).

TABLE 2a. SPATIAL VARIATION IN THE PHYTOPLANKTON COMPOSITION AND ABUNDANCE IN SOME PARTS OF THE LAGOS LAGOON.

\begin{tabular}{|c|c|c|c|c|c|c|c|c|c|c|c|c|}
\hline Stations & 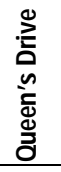 & 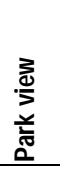 & $\frac{\pi}{0}$ & 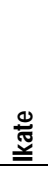 & 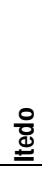 & 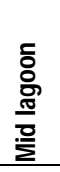 & 劳 & 竞 & $\begin{array}{l}\text { ֻँ } \\
\text { હ }\end{array}$ & 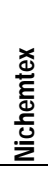 & 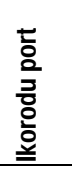 & 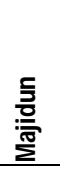 \\
\hline \multicolumn{13}{|l|}{ PHYTOPLANKTON TAXA } \\
\hline \multicolumn{13}{|l|}{ DIVISION - BACILLARIOPHYTA } \\
\hline \multicolumn{13}{|l|}{ CLASS - BACILLARIOPHYCEAE } \\
\hline \multicolumn{13}{|l|}{ ORDER I - CENTRALES } \\
\hline Actinoptychus splendens Ehrenberg & 20 & 25 & - & 5 & - & 5 & - & 10 & 5 & 5 & 15 & 30 \\
\hline Aulacoseira granulata Ehrenberg (Ralfs) & 10 & 20 & 70 & 35 & 50 & 20 & 80 & 75 & 55 & 20 & 75 & 105 \\
\hline Aulacoseira granulata var. angustissima Muller & - & - & 5 & 10 & - & 5 & 10 & 15 & 25 & 20 & 415 & 450 \\
\hline Aulacoseira granulata var. curvata Simon & - & - & - & - & - & 10 & 5 & 15 & 5 & 5 & 5 & 15 \\
\hline \multicolumn{13}{|l|}{ Aulacoseira sp. } \\
\hline Coscinodiscus eccentrius Ehrenberg & 35 & - & 25 & 5 & 5 & - & - & - & - & - & - & - \\
\hline Coscinodiscus radiatus Ehrenberg & - & - & - & - & - & - & - & - & - & - & - & - \\
\hline Cyclotella menighiniana Kutzing & - & - & - & - & 5 & - & - & - & 5 & & 36 & 5 \\
\hline Cyclotella striata (Kutzing) Grunow & - & - & - & - & - & - & - & 5 & - & - & - & \\
\hline Terpsinoe musica (Ehr) Hustedt & - & - & - & - & - & - & - & - & - & - & - & 10 \\
\hline \multicolumn{13}{|l|}{ Order II - PENNALES } \\
\hline $\begin{array}{l}\text { Gyrosigma balticum (Ehr.) Rabenhorst } \\
\text { Gyrosigma balticum (Ehr.) Rabenhorst }\end{array}$ & 15 & 10 & 25 & 5 & - & 10 & - & - & - & - & - & - \\
\hline Navicula mutica Kutzing & & & & & & & & & & & & 15 \\
\hline Pinnularia major (Kutzing) Rabenh & - & - & - & - & - & - & - & 5 & - & 15 & - & 5 \\
\hline Pleurosigma angulatum (Quekett) Wm Smith & 25 & 50 & 10 & - & 5 & - & - & - & - & - & - & - \\
\hline Surirella splendida Wm. Smith & - & - & - & - & - & & - & - & - & 5 & - & 5 \\
\hline Synedra crystallina (Ag) Kutzing & 5 & & 5 & 15 & 5 & - & - & 5 & 15 & 10 & 45 & 15 \\
\hline Synedra sp. & - & - & - & - & - & - & - & - & - & - & - & 10 \\
\hline Synedra ulna (Nitzsch) Ehrenberg & - & - & - & - & - & 10 & - & - & 5 & - & 5 & 15 \\
\hline
\end{tabular}


TABLE 2a. CONT. SPATIAL VARIATION IN THE PHYTOPLANKTON COMPOSITION AND ABUNDANCE IN SOME PARTS OF THE LAGOS LAGOON.

\begin{tabular}{|c|c|c|c|c|c|c|c|c|c|c|c|c|}
\hline & 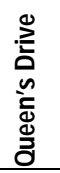 & 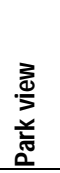 & $\begin{array}{l}\frac{\pi}{0} \\
\frac{0}{\Sigma}\end{array}$ & 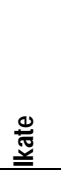 & 욜 & 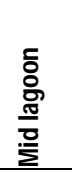 & $\begin{array}{l}\text { ๘ँ } \\
\text { 히 }\end{array}$ & $\stackrel{5}{0}$ & 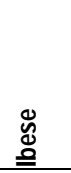 & 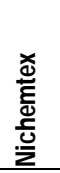 & 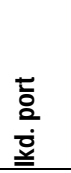 & 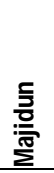 \\
\hline \multicolumn{13}{|l|}{ DIVISION - CYANOPHYTA } \\
\hline \multicolumn{13}{|l|}{ CLASS - CYANOPHYCEAE } \\
\hline \multicolumn{13}{|l|}{ ORDER I - CHROOCOCCALES } \\
\hline Microcystis flos-aquae Kirchner & - & - & - & - & - & 5 & - & 15 & - & - & 20 & 45 \\
\hline Microcystis flos-aquae Kirchner & - & - & - & - & - & - & - & - & - & - & - & 5 \\
\hline \multicolumn{13}{|l|}{ Order II - HORMOGONALES } \\
\hline Oscillatoria chalybea Gomont & - & - & - & - & - & - & - & - & 5 & 25 & - & - \\
\hline Oscillatoria curviceps C.A. Agardh & - & - & - & - & - & - & - & - & - & 15 & 15 & 15 \\
\hline Oscillatoria limnosa Agardh & 15 & 5 & 5 & - & 10 & - & 5 & - & - & 5 & 5 & 5 \\
\hline Oscillatoria tenius Agardh & - & - & - & - & - & 5 & - & - & 15 & - & 5 & - \\
\hline Spirulina platensis Geitler & - & - & - & - & - & - & - & - & - & - & - & 15 \\
\hline \multicolumn{13}{|l|}{ DIVISION - CHLOROPHYTA } \\
\hline \multicolumn{13}{|l|}{ CLASS - CHLOROPHYCEAE } \\
\hline \multicolumn{13}{|l|}{ ORDER I - ULOTHRICALES } \\
\hline Spirogyra africana Fritsch Cruda & - & - & - & - & - & - & - & 5 & - & 5 & - & 25 \\
\hline Spirogyra sp. & - & - & - & - & - & - & - & - & - & - & - & 5 \\
\hline \multicolumn{13}{|l|}{ ORDER II - ZYGNEMATALES } \\
\hline Closterium ehrenbergii Meneghini & - & - & - & - & - & - & - & - & 5 & 35 & 25 & 30 \\
\hline $\begin{array}{l}\text { Closterium moniliferum (Bory.) } \\
\text { Ehrenh. }\end{array}$ & - & - & - & - & - & - & - & - & & 5 & & 5 \\
\hline Gonatozygon sp. & & & & 25 & 5 & 55 & 35 & 50 & & 10 & & 25 \\
\hline \multicolumn{13}{|l|}{ ORDER III - CLADOPHORALES } \\
\hline Cladophora glomerata (L) Kutzing & - & - & - & - & - & - & - & - & - & 5 & - & 25 \\
\hline \multicolumn{13}{|l|}{ DIVISION - EUGLENOPHYTA } \\
\hline \multicolumn{13}{|l|}{ CLASS - EUGLENOPHYCEAE } \\
\hline \multicolumn{13}{|l|}{ ORDER - EUGLENALES } \\
\hline Euglena acus Ehrenberg & - & - & - & - & - & - & - & - & - & 30 & - & 5 \\
\hline Phacus acuminatus Stokes & - & - & - & - & - & - & - & 5 & - & - & - & 5 \\
\hline Phacus curvicauda Swirenko & - & - & - & - & - & - & - & - & - & - & - & \\
\hline Trachelomonas hispida (Perry) Stein & - & - & - & - & - & - & - & - & - & - & 5 & 5 \\
\hline Total species diversity (S) & 7 & 5 & 7 & 7 & 7 & 9 & 5 & 11 & 10 & 16 & 13 & 26 \\
\hline Total phytoplankton abundance (N) & 125 & 110 & 145 & 100 & 85 & 125 & 135 & 205 & 140 & 215 & 671 & 895 \\
\hline
\end{tabular}

Zooplankton Community Structure: For the zooplankton, the Phylum - Crustacea was the dominant phyla recorded for the study. Among the copepods which were the most important group in terms of diversity and abundance, 3 sub-orders namely Calanoida represented by 5 species (Acartia clausii , Acartia discaudata, Acartia tonsa and Paracalanus parvus and Pseudocalanus elongatus), Cyclopoida represented by 5 species (Corycaeus anglicus, Cyclopina longicornis, Cyclops strenus, Cyclops sp. and Oithona plumifera) and Harpaticoida represented by 1 species - Enterpina acutifrons. With regard to the Subclass: Branchiopoda (Cladocerans) they were represented by 4 species namely Bosmina sp., Diaphanosoma excisum, Diaphnia sp. and Penilia avirostris. For the Phylum - Chaetognatha (arrow worms), Sagitta enflata was the sole species recorded. For the Phylum Rotifera (rotifers) 4 species were recorded viz. Brachionus plicatilis, Keratella sp., Lecane bulla and Tetrasiphon hydrocoral (Table 3a).
For the twelve stations studied, diversity (S) ranged between 0 species (Nichemtex) and 9 species (Queens Drive, Moba, Ikorodu port and Majidun). Abundance of individuals per $\mathrm{ml}(\mathrm{N})$ of the samples was between 0 individuals per $\mathrm{ml}$ (Nichemtex) and 120 individuals per $\mathrm{ml}$ (Queens Drive). Whereas Log of Species diversity (Log S) ranged between 0 (Itedo, Oreta and Nichemtex) and 0.95 (Queens Drive, Moba, Ikorodu port and Majidun). Log of phytoplankton abundance $(\log N)$ were between 0 (Nichemtex) and 2.08 (Queens Drive). Furthermore, the values for ShannonWiener Index (Hs) were between 0 (Itedo and Nichemtex) and 0.93 (Ikorodu port). Menhinick Index (D) on the other hand ranged between 0 (Nichemtex) and 1.12 (Ikorodu port). Margalef Index (d) values also ranged between 0 (Itedo, Ofin, Oreta and Nichemtex) and 1.95 (Moba). Equitability Index (j) index values were between 0 (Itedo, Oreta, Ofin and Nichemtex) and 1.0 (Ikate). Simpson's Dominance Index $(\mathrm{C})$ also ranged between 0 (Nichemtex) and 1.00 (Itedo, Oreta and Ofin). (Table 3b). 
TABLE 2b. SPATIAL VARIATION IN PHYTOPLANKTON COMMUNITY COMPOSITION PARAMETERS IN SOME PARTS OF THE LAGOS LAGOON.

\begin{tabular}{|c|c|c|c|c|c|c|c|c|c|c|c|c|}
\hline Bio-indices & 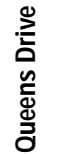 & 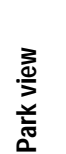 & $\stackrel{\pi}{\frac{\pi}{0}}$ & 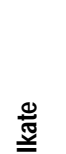 & 을 & 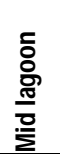 & 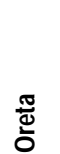 & $\underline{\bar{\partial}}$ & 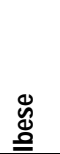 & 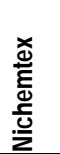 & $\begin{array}{l}\text { 흠 } \\
\text { 흠 } \\
\text { 흠 } \\
\end{array}$ & 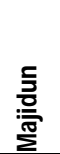 \\
\hline Total species diversity (S) & 7 & 5 & 7 & $\overline{7}$ & $\overline{7}$ & 9 & 5 & 11 & $\overline{10}$ & 16 & $\overline{13}$ & 26 \\
\hline Total phytoplankton abundance (N) & 125 & 110 & 145 & 100 & 85 & 125 & 135 & 205 & 140 & 215 & 671 & 895 \\
\hline Log of Species diversity (Log S) & 0.85 & 0.70 & 0.85 & 0.85 & 0.85 & 0.95 & 0.70 & 1.04 & 1.00 & 1.20 & 1.11 & 1.41 \\
\hline Log of phytoplankton abundance ( $\log N$ ) & 2.10 & 2.04 & 2.16 & 2.00 & 1.93 & 2.10 & 2.13 & 2.31 & 2.15 & 2.33 & 2.83 & 2.95 \\
\hline Shannon-Wiener Index (Hs) & 0.79 & 0.59 & 0.65 & 0.73 & 0.61 & 0.77 & 0.48 & 0.82 & 0.81 & 1.10 & 0.63 & 0.90 \\
\hline Menhinick Index (D) & 0.63 & 0.48 & 0.58 & 0.70 & 0.76 & 0.80 & 0.43 & 0.77 & 0.85 & 1.09 & 0.50 & 0.87 \\
\hline Margalef Index (d) & 1.24 & 0.85 & 1.21 & 1.30 & 1.35 & 1.66 & 0.82 & 1.88 & 1.82 & 2.79 & 1.84 & 3.68 \\
\hline Equitability Index (j) & 0.93 & 0.85 & 0.77 & 0.86 & 0.72 & 0.81 & 0.68 & 0.79 & 0.81 & 0.91 & 0.57 & 0.64 \\
\hline Simpson's Dominance Index (C) & 0.18 & 0.30 & 0.30 & 0.23 & 0.38 & 0.24 & 0.43 & 0.21 & 0.22 & 0.09 & 0.41 & 0.28 \\
\hline
\end{tabular}

TABLE 3a. SPATIAL VARIATION IN THE ZOOPLANKTON COMPOSITION AND ABUNDANCE IN SOME PARTS OF THE LAGOS LAGOON.

\begin{tabular}{|c|c|c|c|c|c|c|c|c|c|c|c|c|}
\hline Stations & 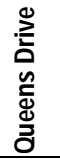 & 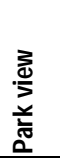 & $\stackrel{\frac{\pi}{0}}{\frac{0}{2}}$ & 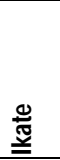 & $\begin{array}{l}\text { 율 } \\
\end{array}$ & 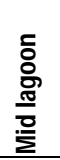 & 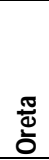 & 咅 & 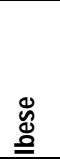 & 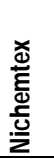 & 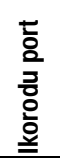 & 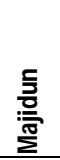 \\
\hline \multicolumn{13}{|l|}{ ZOOPLANKTON TAXA } \\
\hline \multicolumn{13}{|l|}{ PHYLUM-CRUSTACEA } \\
\hline \multicolumn{13}{|l|}{ CLASS - COPEPODA } \\
\hline SUB-ORDER I: CALANOIDA & 25 & 10 & 15 & 5 & - & 5 & - & - & 10 & - & - & - \\
\hline Acartia clausii Giesbrecht & 15 & - & 5 & - & - & - & - & - & - & - & - & - \\
\hline Acartia discaudata Giesbrecht & - & - & - & - & - & - & - & - & - & - & - & - \\
\hline Acartia tonsa Giesbrecht & - & 10 & 5 & - & - & - & - & - & - & - & - & - \\
\hline Paracalanus parvus (Claus) & 5 & 5 & 10 & - & - & 15 & - & 10 & 5 & - & 10 & - \\
\hline Pseudocalanus elongatus (Boeck) & 10 & 5 & 5 & - & - & - & - & - & - & - & - & - \\
\hline \multicolumn{13}{|l|}{ ORDER II - CYCLOPOIDA } \\
\hline Corycaeus anglicus Lubbock & 10 & 5 & - & 5 & 5 & - & - & - & - & - & - & - \\
\hline Cyclopina longicornis Boeck & 5 & - & - & - & - & - & & - & - & - & - & - \\
\hline Cyclops strenus Fisher & & - & - & - & - & - & 5 & - & 5 & - & 10 & 25 \\
\hline Cyclops sp. & - & - & - & - & - & - & & - & - & - & 5 & 5 \\
\hline Oithona plumifera Baird & 15 & 5 & 5 & - & - & - & & - & - & - & - & - \\
\hline \multicolumn{13}{|l|}{ SUB-ORDER III: HARPATICOIDA } \\
\hline Enterpina acutifrons Dana & - & 5 & 5 & - & - & - & - & - & - & - & - & - \\
\hline \multicolumn{13}{|l|}{ SUBCLASS: BRANCHIOPODA } \\
\hline \multicolumn{13}{|l|}{ ORDER : CLADOCERA } \\
\hline \multicolumn{13}{|l|}{ SUB-ORDER: EUCLADOCERA } \\
\hline Bosmina sp. & - & - & - & - & - & - & - & - & 5 & - & 5 & 5 \\
\hline Diaphanosoma excisum (Sar.) & - & - & - & - & - & - & - & - & - & - & 5 & 15 \\
\hline Diaphnia sp. & - & - & - & - & - & - & - & - & - & - & 5 & 10 \\
\hline Penilia avirostris Dana & 25 & 15 & 5 & - & - & - & - & - & - & - & - & - \\
\hline \multicolumn{13}{|l|}{ PHYLUM - CHAETOGNATHA } \\
\hline \multicolumn{13}{|l|}{ ORDER - APHARAGMORPHA } \\
\hline Sagitta enflata Vogt & 10 & - & 5 & - & - & - & - & - & - & - & - & - \\
\hline \multicolumn{13}{|l|}{ PHYLUM: ROTIFERS } \\
\hline \multicolumn{13}{|l|}{ CLASS: MONOGONOTA } \\
\hline \multicolumn{13}{|l|}{ ORDER: PLOIMA } \\
\hline Brachionus plicatilis Muller & - & - & - & - & - & - & - & - & 5 & - & 10 & 15 \\
\hline Keratella sp. & - & - & - & - & - & - & - & - & & - & & 20 \\
\hline Lecane bulla Gosse & - & - & - & - & - & 5 & - & - & 5 & - & 10 & 15 \\
\hline Tetrasiphon hydrocoral Ehrenberg & - & - & - & - & - & & - & - & 5 & - & 5 & 5 \\
\hline Total species diversity (S) & 9 & 8 & 9 & 2 & 1 & 3 & 1 & 1 & 7 & 0 & 9 & 9 \\
\hline Total zooplankton abundance (N) & 120 & 60 & 60 & 10 & 5 & 25 & 5 & 10 & 40 & 0 & 65 & 115 \\
\hline
\end{tabular}




\begin{tabular}{|c|c|c|c|c|c|c|c|c|c|c|c|c|}
\hline & 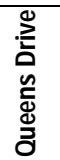 & 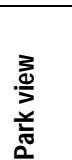 & $\stackrel{\pi}{0}$ & 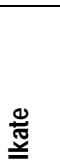 & 윻 & 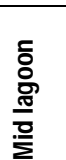 & 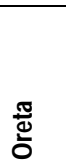 & $\stackrel{\Sigma}{\tilde{L}}$ & 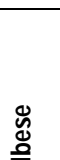 & 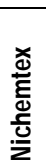 & $\begin{array}{l}\text { 늠 } \\
\text { 픔 } \\
\text { 홓 }\end{array}$ & $\begin{array}{l}\frac{5}{0} \\
\frac{0}{\bar{T}} \\
\sum\end{array}$ \\
\hline Total species diversity (S) & 9 & 8 & 9 & 2 & 1 & 3 & 1 & 1 & 7 & 0 & $\overline{9}$ & 9 \\
\hline Total zooplankton abundance (N) & 120 & 60 & 60 & 10 & 5 & 25 & 5 & 10 & 40 & 0 & 65 & 115 \\
\hline Log of Species diversity (Log S) & 0.95 & 0.90 & 0.95 & 0.30 & 0 & 0.48 & 0 & 0 & 0.85 & 0 & 0.95 & 0.95 \\
\hline Log of zooplankton abundance $(\log N)$ & 2.08 & 1.78 & 1.78 & 1.00 & 0.70 & 1.40 & 0.70 & 1.00 & 1.60 & 0 & 1.81 & 2.06 \\
\hline Shannon-Wiener Index (Hs) & 0.89 & 0.86 & 0.91 & 0.30 & 0 & 0.41 & 0.00 & 0.00 & 0.83 & 0 & 0.93 & 0.89 \\
\hline Menhinick Index (D) & 0.82 & 1.03 & 1.16 & 0.63 & 0.45 & 0.60 & 0.45 & 0.32 & 1.11 & 0 & 1.12 & 0.84 \\
\hline Margalef Index (d) & 1.67 & 1.71 & 1.95 & 0.43 & 0 & 0.62 & 0 & 0 & 1.63 & 0 & 1.92 & 1.69 \\
\hline Equitability Index (j) & 0.94 & 0.95 & 0.95 & 1.00 & 0 & 0.86 & 0 & 0 & 0.98 & 0 & 0.97 & 0.93 \\
\hline Simpson's Dominance Index (C) & 0.14 & 0.15 & 0.14 & 0.50 & 1.00 & 0.44 & 1.00 & 1.00 & 0.16 & 0 & 0.12 & 0.14 \\
\hline
\end{tabular}

TABLE 4a. SPATIAL VARIATION IN THE JUVENILE STAGES COMPOSITION AND ABUNDANCE IN SOME PARTS OF THE LAGOS LAGOON.

\begin{tabular}{|c|c|c|c|c|c|c|c|c|c|c|c|c|}
\hline JUVENILE STAGES & 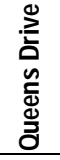 & 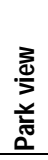 & $\begin{array}{l}\frac{\pi}{0} \\
\sum\end{array}$ & 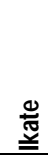 & 윯 & 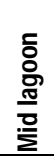 & $\begin{array}{l}\text { 世ँّ } \\
\text { 이 }\end{array}$ & $\stackrel{5}{0}$ & 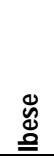 & 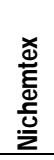 & 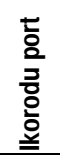 & 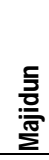 \\
\hline \multicolumn{13}{|l|}{ PHYLUM : ARTHROPODA } \\
\hline \multicolumn{13}{|l|}{ CLASS : CRUSTACEA } \\
\hline Barnacle nauplii larva & 15 & 35 & 20 & 15 & 5 & - & 15 & 5 & 5 & - & 10 & 15 \\
\hline Copepod eggs & 10 & 10 & & 5 & - & 5 & - & - & - & - & - & - \\
\hline Copepods nauplii larva & 25 & 15 & 10 & 5 & - & - & 5 & 10 & 15 & 5 & 10 & 15 \\
\hline Megalop larva & - & 5 & - & - & - & 10 & - & - & 5 & - & - & - \\
\hline Zoea larva of crab & 15 & 10 & 5 & 5 & 10 & 5 & 5 & - & 10 & - & 5 & 15 \\
\hline \multicolumn{13}{|l|}{ PHYLUM: CHORDATA } \\
\hline Fish eggs & 10 & 5 & - & - & 5 & - & - & - & - & - & - & - \\
\hline Fish larva & - & - & 5 & - & - & 5 & - & 5 & - & - & - & - \\
\hline \multicolumn{13}{|l|}{ PHYLUM: MOLLUSCA } \\
\hline Gastropod larva & - & - & - & 10 & - & 15 & 5 & 10 & 5 & - & - & - \\
\hline Bivalve larva & 10 & 5 & - & - & - & 15 & & 5 & 5 & - & - & - \\
\hline \multicolumn{13}{|l|}{ PHYLUM: ANNELIDA } \\
\hline Annelid larva & - & 5 & 5 & - & - & - & - & - & - & - & - & - \\
\hline Forms of juvenile stages & 6 & 8 & 5 & 5 & 3 & 6 & 4 & 5 & 6 & 1 & 3 & 3 \\
\hline Juvenile stage abundance & 85 & 90 & 45 & 40 & 20 & 55 & 30 & 35 & 45 & 5 & 25 & 45 \\
\hline
\end{tabular}

TABLE 4b. SPATIAL VARIATION IN THE JUVENILE STAGES COMMUNITY COMPOSITION PARAMETERS IN SOME PARTS OF THE LAGOS LAGOON.

\begin{tabular}{|c|c|c|c|c|c|c|c|c|c|c|c|c|}
\hline & 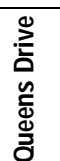 & 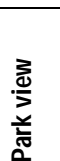 & $\frac{\pi}{0}$ & 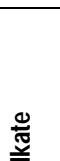 & 윯 & 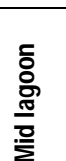 & $\begin{array}{l}\text { कீ } \\
\text { के }\end{array}$ & $\underline{\underline{L}}$ & 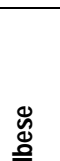 & 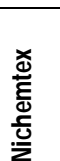 & $\begin{array}{l}\text { 흠 } \\
\text { 믐 } \\
\text { 홓 } \\
\underline{\underline{z}}\end{array}$ & 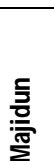 \\
\hline Total species diversity (S) & 6 & 8 & 5 & 5 & 3 & 6 & 4 & 5 & 6 & 1 & 3 & 3 \\
\hline Total phytoplankton abundance (N) & 85 & 90 & 45 & 40 & 20 & 55 & 30 & 35 & 45 & 5 & 25 & 45 \\
\hline Log of Species diversity (Log S) & 0.78 & 0.90 & 0.70 & 0.70 & 0.48 & 0.78 & 0.60 & 0.70 & 0.78 & 0 & 0.48 & 0.48 \\
\hline Log of phytoplankton abundance $(\log N)$ & 1.93 & 1.95 & 1.65 & 1.60 & 1.30 & 1.74 & 1.48 & 1.54 & 1.65 & 0.70 & 1.40 & 1.65 \\
\hline Shannon-Wiener Index (Hs) & 0.75 & 0.78 & 0.62 & 0.65 & 0.45 & 0.73 & 0.54 & 0.67 & 0.73 & 0 & 0.46 & 0.48 \\
\hline Menhinick Index (D) & 0.65 & 0.84 & 0.75 & 0.79 & 0.67 & 0.81 & 0.73 & 0.85 & 0.89 & 0.45 & 0.60 & 0.45 \\
\hline Margalef Index (d) & 1.13 & 1.56 & 1.05 & 1.08 & 0.67 & 1.25 & 0.88 & 1.13 & 1.31 & 0 & 0.62 & 0.53 \\
\hline Equitability Index (j) & 0.96 & 0.86 & 0.89 & 0.93 & 0.95 & 0.93 & 0.90 & 0.96 & 0.94 & 0 & 0.96 & 1.00 \\
\hline Simpson's Dominance Index (C) & 0.19 & 0.22 & 0.28 & 0.25 & 0.38 & 0.21 & 0.33 & 0.22 & 0.21 & 1.00 & 0.36 & 0.33 \\
\hline
\end{tabular}


Juvenile Forms: With regard to juvenile stages the Phylum Arthropoda was represented by barnacle nauplii, copepod eggs, copepods nauplii, megalop larvae and zoea larvae of crab. For the Phylum Chordata, fish eggs and fish larvae were represented. For the Phylum Mollusca, Gastropod and bivalve larvae were represented and with regard to the Phylum Annelida, annelid larvae were represented. The crustacean juvenile forms were the more important form in terms of diversity and occurrence. Barnacle nauplii were the most frequent occurring form of the lot (Table 4a). Juvenile forms community composition parameters for the twelve stations studied are shown in Table $4 \mathrm{~b}$

\section{DISCUSSION}

The highest air temperature $\left(30.5^{\circ} \mathrm{C}\right)$ and water temperature $\left(29.5^{\circ} \mathrm{C}\right)$ were recorded at Ibese sampling station. Surface water temperature values were high $\left(28^{\circ} \mathrm{C}-29.5^{\circ} \mathrm{C}\right)$ in all the sampling stations. Majidun had the lowest dissolved oxygen values $(5.2 \mathrm{mg} / \mathrm{l})$ when compared with other stations. This value is just a little above the minimum WHO standard of $5 \mathrm{mg} / \mathrm{l}$ required for water quality assessment. The water samples were very turbid in all the stations. The period of sampling was a rainy season and particulate matters brought into the lagoon by surface run-off and flood must have caused high turbidity. Zero salinity value was recorded for all the stations and this implies a freshwater condition. This is indicative of the period of sampling, a typical rainy season.

According to Nwankwo (1996) the dynamic interplay between freshwater inflow and tidal seawater incursion determine the Lagos lagoon environment from year to year. For instance In the wet season according to Onyema et al., (2003), there is increased river inflow which creates freshwater and low brackish water conditions in various parts of the lagoon. For the present study, salinity levels were low at all the stations samples. This is likely due to freshwater inflow and reduced tidal incursion (Hill \& Webb, 1958). Onyema (2007) observed a direct relation between the seasonal bimodal rainfall pattern, the environmental and biotal gradient in the Lagos lagoon.

With regards to the plankton spectrum the diatoms were clearly the dominant phytoplankton form where as the copepods particularly the calanoid forms were the more important forms for the zooplankton. Onyema et al., (2003) observed that whereas diatoms dominate the phytoplankton population, copepods were prominent members of the zooplankton population of the Lagos lagoon. Nwankwo (1996) had earlier reported high diatom production in the Lagos lagoon and attributed the numerous pennate forms to the tidal induced mixing of the phytobenthic forms and the plankton.

The juvenile forms encounter in the study were also ecologically important. The Lagos lagoon has been described by authors as a nursery ground for an array of aquatic biota. For this study for instance a total of 10 juvenile stages were recorded. This is more than the numbers recorded by Akpata et al., 1993, Onyema et al., $(2003,2007)$ and Onyema \& Ojo (2008). This may reflect that the Lagos lagoon even in the wet season still serves as a nursery and breeding ground for juveniles as evident by the presence of an array of juvenile stages. Owing to the distributive rainfall pattern in southwestern Nigeria there exists four ecologically important periods in our coastal waters (Nwankwo, 2004). These are the dry months (Jan -March/ April) when high salinity is experienced in the coastal waters and lagoons; April/May, when the salinity drops drastically causing a stress condition and a resultant loss of biodiversity arising from the death of marine biota that invade the coastal water in the dry high salinity months. At this time (April/May) only opportunistic forms such as Gomphosphaeria aponina, Amphora coffeaformis abound. There are the wet months (June - Nov.) when the coastal lagoons are fresh and December when the salinity rises sharply, excluding freshwater forms. This may explain why there was few brackish water or estuarine species recorded. Additionally a significant number of the species recorded in this study were freshwater species. For instance Aulacoseira granulate, Aulacoseira granulata var. angustissima, Aulacoseira granulata var. curvata, Microcystis flos-aquae, Microcystis aureginosa, Spirogyra Africana, Closterium ehrenbergii, Gonatozygon sp. and Euglena acus among others have been reported by a number of authors (Kadiri, 1999, Nwankwo, 1988, Onyema, 2008) as fresh water species. Few authors are of the view that the most productive period in the coastal waters of south western Nigeria occurs after the second rainfall period (October or November) and that plankton diversity in the Lagos lagoon increases towards the harbour.

\section{ACKNOWLEDGEMENT}

We appreciate the Director of Biological Oceanography of the Nigerian Institute for Oceanography and Marine Research and Dr. E. O. Oyewo for their guidance and encouragement. We thank the staff of the Department of Marine Sciences, University of Lagos for their collaborative spirit.

\section{REFERENCES}

Ajao, E. A. (1996). Review of the state of pollution of the Lagos lagoon. NIOMR Technical Paper No. 106.

Akpata, T. V. I.; Oyenekan, J. A. \& Nwankwo, D. I. (1993). Impact of organic pollution on the Bacterial, Plankton and Benthic Population of Lagos Lagoon, Nigeria. International Journal of Ecology and Environmental Science. 19: 73-82.

Barnes, R. S. K., Calow, P. \& Olive, P. J. W. (1993). The invertebrates: a new synthesis. Blackwell Scientific Publications. London.

Edokpayi, C. A. \& Nkwoji, J. A. (2007). Annual changes in the physico-chemical and macrobenthic invertebrate characteristics of the Lagos lagoon sewage dump site at Iddo, Southern Nigeria. Ecology, Enironment \& Conservation 13(1): 13-18.

Food and Agricultural Organisat ion(1969). Fisheries Survey in the Western and Mid-Western Regions of Nigeria. FAO/Sf: 74/NIR 6.

Hill, M. B. \& Webb, J. E. (1958). The ecology of Lagos lagoon II. The topography and physical features of the Lagos harbour and Lagos lagoon. Philosophical Transaction of Royal Society, London. 241:307-417.

Kadiri, M. O. (1999). Phytoplankton distribution in some coastal waters of Nigeria. Nigeria Journal of Botany.12 (1): 51 - 62.

Nwankwo, D. I. (1986). Phytoplankton of a sewage disposal site in Lagos lagoon, Nigeria 1. The algae. Nigerian Journal of Biological Sciences. 1: 89-91.

Nwankwo, D. I. (1988). A preliminary checklist of planktonic algae in Lagos lagoon Nigeria. Nigeria. Journal of Botanica I Applied Sciences.. 2: 73-85.

Nwankwo, D. I. (1990). Contribution to the Diatom flora of Nigeria. Diatoms of Lagos lagoon and the adjacent sea. Nigerian Journal of Botany. 3: 53-70.

Nwankwo, D. I. (1995). Euglenoids of some Polluted Storm-water Channels in Lagos, Nigeria. Tropical Freshwater Biology 4: 29 - 39. 
Nwankwo, D. I. (1998). The influence of sawmill wood wastes on diatom population at Oko-baba, Lagos, Nigeria. Nigerian journal of Botany 11: 15-24.

Nwankwo, D. I. \& Akinsoji (1988). Periphyton Algae of eutrophic Creek and their possible use as Indicator. Nigerian Journal of Botany 1: 96-105.

Ogbeibu, A. E. (2005). Biostatistics: A practical approach to reseach and data handling. Mindex Publishing Company limited, Benin city, Nigeria.

Olaniyan, C. I. O. (1969). The seasonal variation in the hydrology and total plankton of the lagoons of South West-Nigeria. Nigerian Journal of Science. 3(2):101-119.

Olaniyan, C. I. O. (1975). An introduction to West African Ecology. Heinemann Education Books Ltd., London.

Onyema, I. C., Otudeko, O. G. and Nwankwo, D. I. (2003). The distribution and composition of plankton around a sewage disposal site at Iddo, Nigeria. Journal of Scientific Research Development.7: $11-26$

Onyema, I. C. (2007). Mudflat microalgae of a tropical bay in Lagos, Nigeria. Asian Journal of Microbiology, Biotechnology and Environmental Sciences. 9 (4): 877 - 883.

Onyema, 1. C., Okpara, C. U., Ogbebor, C. I. Otudeko, O. \& Nwankwo, D. I. (2007). Comparative studies of the water chemistry characteristics and temporal plankton variations at two polluted sites along the Lagos lagoon, Nigeria. Ecology, Environment and conservation. 13: $1-12$.
Onyema, I. C. \& Ojo, A. A. (2008). The zooplankton dynamics and chlorophyll a concentration of a tropical tidal creek in relation to water quality indices. Life Science Journal. 5 (4): 7-14

Oyewo, E. O. (1998). Industrial sources and distribution of heavy metals in Lagos lagoon and their biological effects on estuarine animals. Ph.D Thesis, University of Lagos, Nigeria. 279pp.

Rosowski, J. R. (2003). Photosynthetic Euglenoids. In: Freshwater Algae of North America. Ecology and Classification, Wehr, J.D. and Sheath, R.G. (Eds). Academic Press, New York.

Siver, P. A. (2003). Synurophyte algae. In: Freshwater Algae of North America. Ecology and Classification. Wehr, J.D. and Sheath, R.G. (Eds). Academic Press, New York.

Shannon, C. E. \& Weiner, W. (1963). The mathematical theory of communication. Urban University Illinois Press.

Vanlandingham, S. L. 1982. Guide to the identification and environmental requirements and pollution tolerance of freshwater blue-green algae (cyanophyta). U.S. Environmental Protection Agency, EPA - 60 .

Webb, J. E. (1958a). The Ecology of Lagos lagoon. 1: The lagoons of the Guinea Coast. Philosophical Transaction Royal Society London. Ser B 241-283.

Waife, G. \& Frid, C. L. J. (2001). Marine zooplankton of West Africa. Marine Biodiversity Capacity Building in the West African Subregion. Darwin Initiative Reports 5, Ref. 162/7/45/. 120pp

Wimpenny, R. S. (1966). The plankton of the sea. Faber and Faber Limited, London 